\title{
Sweeping Up Dying Cells during Tissue Injury
}

\author{
Yohei Arai ${ }^{a, b}$ Yusuke Yamaoka ${ }^{a, c}$ Sho Morioka ${ }^{a, d}$ \\ aDivision of Nephrology and CIIR, Department of Medicine, University of Virginia Health System, Charlottesville, \\ VA, USA; ${ }^{b}$ Department of Nephrology, Graduate School of Medicine, Tokyo Medical and Dental University, Tokyo,

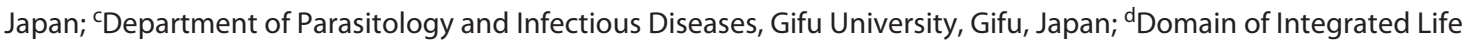 \\ Systems, Center for Highly Advanced Integration of Nano and Life Sciences, Gifu University, Gifu, Japan
}

\section{Keywords}

Acute kidney injury - Apoptosis - Efferocytosis .

Phagocytosis · Programmed cell death

\begin{abstract}
Various forms of cell death have been identified, and billions of cells die during development and daily in adult organisms. Clearing dead cells and associated cellular debris is an integral part of tissue homeostasis. While diverse types of phagocytes remove various forms of dying cells during acute kidney injury (AKI), it remains unknown whether boosting removal of a specific form of dying cell would provide a benefit and which cell type should be targeted for phagocytosis-mediated therapy. As there is a lack of viable strategies for the prevention and treatment of AKI, novel therapies and innovative approaches are required. There is a strong demand on developing and analyzing novel models to boost, monitor, and stop phagocytosis of dying cells.
\end{abstract}

(c) 2021 S. Karger AG, Basel

Contribution from the AKI and CRRT 2021 Symposium at the 26th International Conference on Advances in Critical Care Nephrology, A Virtual/Hybrid Event from San Diego, CA, USA, February 28-March 5, 2021. This symposium was supported in part by the NIDDK funded University of Alabama at Birmingham-University of California San Diego O'Brien Center for Acute Kidney Injury Research (P30DK079337).

\section{Introduction}

Recent studies have focused on "how cell death affects tissue injury." Various forms of programmed cell death (PCD) have been implicated in disease pathologies, and massive amounts of dead cells are rapidly generated during tissue injury. The adequate clearance of dying cells by phagocytic cells is important for maintaining routine tissue homeostasis as well as tissue repair during injury resolution. Dying cells have to be quickly and promptly eliminated by professional and nonprofessional phagocytes, such as fibroblasts and epithelial cells. Dying cells, if not efficiently cleared, release damage-associated molecular patterns (DAMPs), exacerbate inflammation, and perturb the resolution of tissue injury. Recent studies revealed that dead cell or debris removal plays an important role in hampering various diseases, suggesting that dead cell clearance could be a novel therapeutic target. Defective clearance of dying cells is associated with exacerbation of acute kidney injury (AKI); however, whether targeting a specific form of dying cell for removal would be beneficial has not been determined yet. It is also imperative to explore whether boosting cell clearance can improve kidney pathology and function during/after injury. karger@karger.com www.karger.com/nef (c) 2021 S. Karger AG, Basel

Karger'
Correspondence to:

Sho Morioka, sm9ss@ virginia.edu 


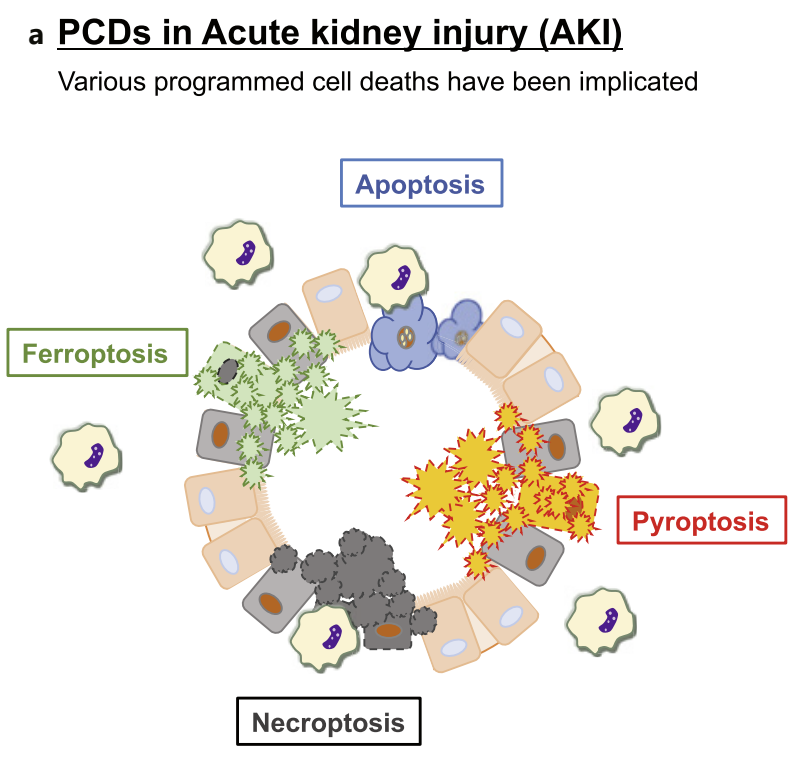

b Dying cell clearance in tissue repair

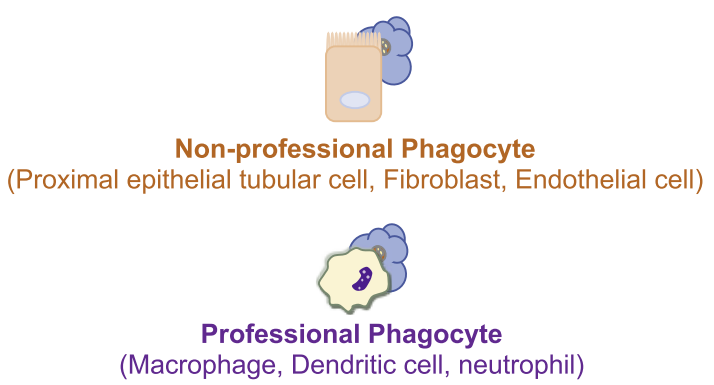

c Future direction

\begin{tabular}{|l|}
\hline Boosting cell clearance can be a novel therapeutic strategy \\
- Removing apoptotic cells or other type of dying cells \\
facilitates resolution of AKI? \\
- Which type of phagocytes should be targeted \\
for boosting clearance in tissue repair? \\
$\rightarrow$ Models to boost, monitor and inhibit phagocytosis \\
will bring new insight into programmed cell death \\
and clearance in tissue injury.
\end{tabular}

Fig. 1. Schematic summary of sweeping up dying cells during kidney injury. a Various forms of PCD have been reported in a number of pathogenesis. During AKI, apoptosis, necroptosis, ferroptosis, and pyroptosis are implicated dependent on the cause of injury [7]. $\mathbf{b}$ The prompt removal of dying cells by professional and nonprofessional phagocytes facilitates resolution of tissue injury [8-10]. c The phagocytosis of dying cells during AKI can be a new

\section{Programmed Cell Death in Tissue Injury}

PCD is a final cellular decision to eliminate unfunctional cells and maintain organismic homeostasis [1]. Various forms of PCD have been identified including apoptosis, necroptosis, pyroptosis, ferroptosis, entotic cell death, and autophagy-dependent cell death, which are activated by specific conditions. PCD is strictly regulated by molecularly well-defined effector mechanisms and structured signaling pathways, and they are evolutionarily conserved processes in both invertebrates and vertebrates [2-4]. DNA lesions and ER stress caused by cellular damage typically unleash proapoptotic BCL-2 family proteins, resulting in intrinsic apoptosis via proapoptotic caspase activation. Moreover, the death-receptor ligands, such as FasL and TNFa, produced in injured tissue induce extrinsic apoptosis. On the other hand, various noxious stimuli acting through receptors including these death receptors also induce necroptosis via the therapeutic direction $[14,15]$. Next exciting steps include whether boosting removal of specific form of dying cells would provide a benefit during AKI and which cell type should be targeted for phagocytosis-mediated therapy. There is a strong demand on developing novel models to boost, monitor, and inhibit phagocytosis of dying cells. AKI, acute kidney injury; PCD, programmed cell death.

RIPK1-RIPK3-MLKL pathway when apoptotic pathways are inhibited. Activation of the inflammasome caused by inflammatory disorders triggers pyroptosis via caspase-1 activation. The increase of cytotoxic reactive oxygen species produced via iron-dependent lipid peroxidation during tissue injury causes ferroptosis regulated by glutathione peroxidase 4. PCD is involved in fundamental physiological processes such as normal cell turnover and proper tissue development, but also, dysregulated PCD is implicated in various pathologies. Understanding which form of PCD is involved in each pathological condition and how modulation of PCD affects the outcome are particularly important for developing new therapeutic directions. During AKI, apoptosis, necroptosis, ferroptosis, and pyroptosis are implicated depending on the cause of injury [5] (Fig. 1a). For instance, apoptosis and necroptosis are independently involved in pathogenesis of folic acid-induced AKI. Ferroptosis dominates rhabdomyolysis-induced AKI. Pyroptosis often occurs in contrast-in- 
duced AKI. Moreover, a single type of PCDs in the epithelial layer of the tubules can also initiate the chain reaction of various types of PCDs [5].

\section{Removal of Dying Cells and Tissue Homeostasis}

Apoptosis can be distinguished by morphologic and biochemical characteristics from other forms of PCD leading to primary necrosis. While apoptosis is an immunologically silent form of PCD displaying cell membrane blebbing and shrinkage without the discharge of intracellular contents, necrosis results in rapid membrane rupture and the exposure of intracellular DAMPs, provoking further inflammation during tissue injury [4]. The prompt removal of dying cells by phagocytic cells is important for maintaining tissue homeostasis [6-8]. The failure to clear apoptotic cells results in secondary necrosis and the accumulation of necrotic cells finally promotes tissue inflammation and exacerbates organ damage. Apoptotic cells quickly expose phosphatidylserine as an "eat me" signal on their cell membrane in a caspase-dependent manner, leading to their engulfment by phagocytic cells (i.e., efferocytosis). Necrotic cells can be disintegrated into small debris and engulfed by phagocytic cells like micropinocytosis, being recognized "eat-me" signals such as deposition of complements and opsonization by immune globulin [9]. In most tissues, dying cells can be phagocytosed by either professional or nonprofessional phagocytic cells (Fig. 1b). The professional phagocytic cells include many types of white blood cells such as tissue-resident macrophages, immature dendritic cells, and neutrophils. These phagocytes have the capacity to take in dying cells quickly and process plural corpses in a row. In contrast, the nonprofessional phagocytic cells can be epithelial cells, endothelial cells, fibroblasts, or other stromal cells, depending on the tissue type. These nonprofessional phagocytes generally ingest neighboring dying cells at a slower pace and commonly have a lower capacity to take up plural corpses in a row. Despite the actions of phagocytes to remove apoptotic cells, excessive dying cells remaining to be cleared have been linked to disease progression. Cardiovascular disease and inflammatory bowel disease are well-linked to defects in apoptotic cell clearance and are the major causes of mortality worldwide $[10,11]$. Defective clearance of dying cells causes accumulation of dead cells, releasing pro-inflammatory intracellular contents such as DAMPs, exacerbating tissue inflammation. The removal of dying cells is indispensable to tissue homeostasis. Thus, sweeping up dying

Efferocytosis during Tissue Injury cells can restore tissue homeostasis following injury. However, whether we can boost dying-cell clearance for health benefit remains to be explored.

\section{Removal of Dying Cells during AKI}

The prevalence of kidney disease is increasing worldwide. In the USA, > 40 million people have kidney disease, of whom nearly 1 million have reached end-stage renal disease requiring dialysis or transplantation. Of note, the incidence of AKI is also increasing throughout the world. AKI is associated with high mortality, morbidity, risk of chronic kidney disease, and end-stage renal disease. As there is a lack of viable strategies for the prevention and treatment of AKI, novel therapies and innovative approaches are required [12]. A large number of dying cells are rapidly generated during AKI [5]. PCD in AKI occurs especially in the proximal tubules at the renal corticomedullary junction. AKI causes the partial or diffuse detachment of dying proximal epithelial tubular cells with loss of the brush border. These dying cells produce excessive inflammatory mediators such as DAMPs and attract infiltrating immune cells, further exacerbating kidney injury. Furthermore, the inner lumen of injured tubules is physically obstructed by dead-cell debris. Finally, these events together cause renal dysfunction. Recent studies revealed that specific molecules involved in recognition of necrotic cells by phagocytes played an important role in resolution of AKI. The apoptosis inhibitor of macrophage protein accumulates on necrotic cell debris and enhances the phagocytic removal of the debris by the proximal epithelial tubular cells mediated by kidney injury molecule-1 [13, 14]. The macrophage-inducible C-type lectin expressed on infiltrating macrophages senses dying renal tubular cells expressing $\beta$-glucosylceramide and inhibits the phagocytic activity of macrophages to induce sustained inflammation [15]. These findings suggest that the phagocytosis of necrotic cells during kidney injury can be beneficial. The next exciting steps include elucidating whether boosting removal of a specific form of dying cell (e.g., apoptosis) would provide a benefit during AKI and which cell type should be targeted for phagocytosismediated therapy. There is a strong demand on developing novel models to boost, monitor, and stop phagocytosis of dying cells (Fig. 1c). These models make it possible to explore how regulating specific dying-cell clearance affects kidney pathology and function during injury and to evaluate the outcome of AKI with specific modification of 1 type of phagocyte. 


\section{Concluding Remarks}

Elegant studies have revealed that various types of PCD have been involved in numerous disease pathologies. Detecting specific types of PCD is important, yet we often dismiss the importance of nondetected forms of PCD as their clearance efficiency may differ, or they may rapidly transition to other types of death (e.g., apoptosis to necrosis). Understanding which type of PCD is involved, and moreover, if the dying cells can be removed for beneficial purposes are extremely fascinating. Also, therapeutic strategies to inhibit PCD pathways during tissue injury might be one direction however as inhibition of one death pathway often leads to activation of an alternative mode of PCD, or the accumulation of damaged and nonfunctional cells, this strategy may be less beneficial than boosting clearance. Thus, defining mechanisms of dying cells during homeostatic cell turnover and tissue injury is at an exciting phase, and insights into potential points of therapeutic intervention could have implications for many human diseases.

\section{Acknowledgements}

The research is supported by grants from the Mishima-Kaiun Memorial Foundation, The Kanae Foundation for the Promotion of Medical Science, Yamada Bee Company Inc., NIH/NIDDK K01 DK123497, the ASN Foundation for Kidney Research Transition to Independence Grant, and O'Brien Center Grant Award Number P30 DK079337 (S.M.). Y.A. is supported by the Uehara Memorial Foundation Research Fellowship, and Y.Y. is supported by Japan Society for the Promotion of Science Research Fellowship for Young Scientists.

\section{Conflict of Interest Statement}

The authors report no conflict of interest.

\section{References}

1 Mihaly SR, Ninomiya-Tsuji J, Morioka S. TAK1 control of cell death. Cell Death Differ. 2014;21(11):1667-76.

2 Morioka S, Broglie P, Omori E, Ikeda Y, Takaesu G, Matsumoto K, et al. TAK1 kinase switches cell fate from apoptosis to necrosis following TNF stimulation. J Cell Biol. 2014; 204(4):607-23

3 Mihaly SR, Sakamachi Y, Ninomiya-Tsuji J, Morioka S. Noncanonical cell death program independent of caspase activation cascade and necroptotic modules is elicited by loss of TGF $\beta$-activated kinase 1. Sci Rep. 2017 Jun 7; $7(1): 2918$.

4 Kim EH, Wong SW, Martinez J. Programmed necrosis and disease:we interrupt your regular programming to bring you necroinflammation. Cell Death Differ. 2019;26(1):25-40.

5 Belavgeni A, Meyer C, Stumpf J, Hugo C, Linkermann A. Ferroptosis and necroptosis in the kidney. Cell Chem Biol. 2020;27(4): $448-62$.
6 Morioka S, Perry JSA, Raymond MH, Medina $\mathrm{CB}, \mathrm{Zhu} \mathrm{Y}$, Zhao L, et al. Efferocytosis induces a novel SLC program to promote glucose uptake and lactate release. Nature. 2018; 563(7733):714-8

7 Morioka S, Maueröder C, Ravichandran KS. Living on the edge: efferocytosis at the interface of homeostasis and pathology. Immunity. 2019;50(5):1149-62.

8 Boada-Romero E, Martinez J, Heckmann BL, Green DR. The clearance of dead cells by efferocytosis. Nat Rev Mol Cell Biol. 2020;21(7): 398-414.

9 Westman J, Grinstein S, Marques PE. Phagocytosis of necrotic debris at sites of injury and inflammation. Front Immunol. 2019 Jan 9; 10: 3030 .

10 Moore KJ, Tabas I. Macrophages in the pathogenesis of atherosclerosis. Cell. 2011;145(3): $341-55$.
11 Lee CS, Penberthy KK, Wheeler KM, Juncadella IJ, Vandenabeele P, Lysiak JJ, et al. Boosting apoptotic cell clearance by colonic epithelial cells attenuates inflammation in vivo. Immunity. 2016;44(4):807-20.

12 Okusa MD, Rosin DL, Tracey KJ. Targeting neural reflex circuits in immunity to treat kidney disease. Nat Rev Nephrol. 2017;13(11): 669-80.

13 Arai S, Kitada K, Yamazaki T, Takai R, Zhang $\mathrm{X}$, Tsugawa Y, et al. Apoptosis inhibitor of macrophage protein enhances intraluminal debris clearance and ameliorates acute kidney injury in mice. Nat Med. 2016;22(2):183-93.

14 Ichimura T, Asseldonk EJ, Humphreys BD, Gunaratnam L, Duffield JS, Bonventre JV. Kidney injury molecule-1 is a phosphatidylserine receptor that confers a phagocytic phenotype on epithelial cells. J Clin Invest. 2008; 118(5):1657-68.

15 Tanaka M, Saka-Tanaka M, Ochi K, Fujieda K, Sugiura Y, Miyamoto T, et al. C-type lectin Mincle mediates cell death-triggered inflammation in acute kidney injury. J Exp Med. 2020;217(11):e20192230. 